\title{
AN AREA INEQUALITY FOR ELLIPSES INSCRIBED IN QUADRILATERALS
}

\section{Alan HORWITZ}

Abstract. If $E$ is any ellipse inscribed in a convex quadrilateral, $Ð$, then we prove that $\frac{\operatorname{Area}(E)}{\operatorname{Area}(\nexists)} \leqslant$ $\frac{\pi}{4}$, and equality holds if and only if $Ð$ is a parallelogram and $E$ is tangent to the sides of $Ð$ at the midpoints. We also prove that the foci of the unique ellipse of maximal area inscribed in a parallelogram, $Ð$, lie on the orthogonal least squares line for the vertices of $Ð$. This does not hold in general for convex quadrilaterals.

Mathematics subject classification (2010): 52A38.

Keywords and phrases: Area inequality; elipse; quadrilaterals.

\section{REFERENCES}

[1] G. D. Chakerian, A Distorted View of Geometry, MAA, Mathematical Plums, Washington, DC, 1979, $130-150$.

[2] Alan Horwitz, Ellipses of maximal area and of minimal eccentricity inscribed in a convex quadrilateral, Australian Journal of Mathematical Analysis and Applications, 2 (2005), 1-12.

[3] Alan Horwitz, Ellipses inscribed in parallelograms, submitted to the Australian Journal of Mathematical Analysis and Applications.

[4] D. Kalman, An elementary proof of Marden's theorem, American Mathematical Monthly, 115 (2008), 330-338.

[5] D. Minda And S. Phelps, Triangles, Ellipses, and Cubic Polynomials, American Mathematical Monthly, 115 (2008), 679-689.

[6] ERIC W. Weisstein, Ellipse, From MathWorld-A Wolfram Web Resource. http://mathworld. wolfram.com/Ellipse.html 